\title{
Long-term effect of metformin on blood glucose control in non-obese patients with type 2 diabetes mellitus
}

\author{
Hiroyuki Ito ${ }^{*}$, Hidenori Ishida, Yuichiro Takeuchi, Shinichi Antoku, Mariko Abe, Mizuo Mifune, Michiko Togane
}

\begin{abstract}
Background: We aimed to investigate the long-term effect of metformin on the blood glucose control in non-obese patients with type 2 diabetes mellitus.

Methods: A retrospective study was performed in 213 patients with type 2 diabetes mellitus under the administration of metformin for more than one year. The clinical parameters were investigated for 3 years. The obese and non-obese individuals were defined as a body mass index (BMI) of $25 \mathrm{~kg} / \mathrm{m}^{2}$ or over $(n=105)$ and a BMl of less than $25 \mathrm{~kg} / \mathrm{m}^{2}(n=108)$, respectively.

Results: $\mathrm{HbA} 1 \mathrm{c}$ levels were significantly decreased compared with those at the baseline time. The course of HbA1c was similar between the non-obese and the obese groups, while the dose of metformin required to control blood glucose was significantly lower in the non-obese group than in the obese group. The reductions in HbA1c were $1.2 \%$ and $1.1 \%$ at 12 months, $0.9 \%$ and $0.9 \%$ at 24 months, and $0.8 \%$ and $1.0 \%$ at 36 months in the non-obese and obese groups, respectively. BMI did not change during the observation periods. Approximately half of all patients required no additional antidiabetic agents or a reduction in other treatments after the initiation of metformin in either of the two groups.

Conclusions: The present study demonstrated the long-term beneficial effect of metformin in non-obese (BMI < $25 \mathrm{~kg} / \mathrm{m}^{2}$ ) diabetic patients. This effect appears to be maintained even after the observation period of this study, because metformin was limited to a relatively low dose in the non-obese group and the observed worsening in glycemic control over time can probably be attenuated by increasing the dose of metformin.
\end{abstract}

\section{Background}

Metformin, one of the biguanide agents, has been recommended for the treatment of patients with type 2 diabetes mellitus according to the consensus algorithm published by the European Association for the Study of Diabetes (EASD) and American Diabetes Association (ADA), because it is economical, induces less weight gain and does not cause hypoglycemic attacks, in addition to its glucose-lowering effect [1]. The UK Prospective Diabetes Study (UKPDS) demonstrated that metformin is as effective as sulfonylurea to control the blood glucose levels of obese patients with type 2 diabetes mellitus. Metformin yielded a stable patient body

\footnotetext{
* Correspondence: ito@edogawa.or.jp

* Correspondence: ito@edogawa.or.jp Tokyo 133-0052, Japan
}

(c) 2010 Ito et al; licensee BioMed Central Ltd. This is an Open Access article distributed under the terms of the Creative Commons Attribution License (http://creativecommons.org/licenses/by/2.0), which permits unrestricted use, distribution, and reproduction in any medium, provided the original work is properly cited.

weight, cardiovascular protection and a better patient survival rate compared with sulfonylurea or insulin therapy $[2,3]$. Therefore, metformin is now accepted as the first-line drug for the type 2 diabetic obese patients.

The body mass index (BMI) and body fat percent are different between Asians and Caucasians. The BMI is 3 - 4 units lower in the general population in Asian compared to Caucasians [4]. It is similar in Japanese diabetic patients, for example, the mean BMI was 22.9 $\mathrm{kg} / \mathrm{m}^{2}$ in men and $23.4 \mathrm{~kg} / \mathrm{m}^{2}$ in women in the Japan Diabetes Complications Study (JDCS), which was a nationwide multi-center prospective study of type 2 diabetic patients [5]. Therefore, the strategy for treating non-obese patients is considered to be important in the treatment of type 2 diabetes mellitus for Asians, including Japanese. 
Although it has been reported that metformin is effective for non-obese diabetic individuals, the observation periods were relatively short, usually less than one year [6-12]. Because the effects of oral hypoglycemic agents (OHAs), including metformin, and insulin are gradually reduced as the treatment periods increase $[2,13]$, the observation of metformin over a longer duration is necessary.

In the current study, we aimed to investigate the longterm effects of metformin on blood glucose control in non-obese Japanese patients with type 2 diabetes mellitus.

\section{Patients and Methods}

Three hundred ninety-five subjects who received metformin were conducted to be eligible for this study among 1371 patients with type 2 diabetes mellitus being treated in the Department of Diabetes, Metabolism and Kidney Diseases of Edogawa Hospital, Tokyo, Japan between April 2008 and March 2009 (Figure 1). Any individuals who had stopped visiting our department by changing the hospital or for other unspecified reasons for less than 1 year $(n=129)$ or who discontinued metformin within
1 year after the initiation because of side effects $(n=30)$, such as gastrointestinal symptoms and liver injury, were excluded from this study. The patients with an uncertain metformin treatment start date or without clinical characteristics at the baseline (before initiating metformin therapy) time were also excluded from the present series $(n=23)$. Finally, a retrospective study was performed in a population of 213 patients with type 2 diabetes mellitus under the consecutive administration of metformin for more than 1 year. The clinical parameters, including $\mathrm{BMI}, \mathrm{HbA} 1 \mathrm{c}$ level and the history of medication were investigated from the baseline (the initial date of metformin therapy) for 3 years.

All of the indications, doses and discontinuation of metformin were guided by each patient's physician. Although the optimization of the treatment, such as a dose increase of metformin, an addition/reduction of the other OHAs and an introduction of insulin therapy, was also judged by the chief physician, it was performed if the HbA1c levels remained higher than $7 \%$ or less than $5.8 \%$ for $2-3$ months [14].

The obese and non-obese individuals were defined as having a $\mathrm{BMI} \geq 25 \mathrm{~kg} / \mathrm{m}^{2}$ and a $\mathrm{BMI}<25 \mathrm{~kg} / \mathrm{m}^{2}$,

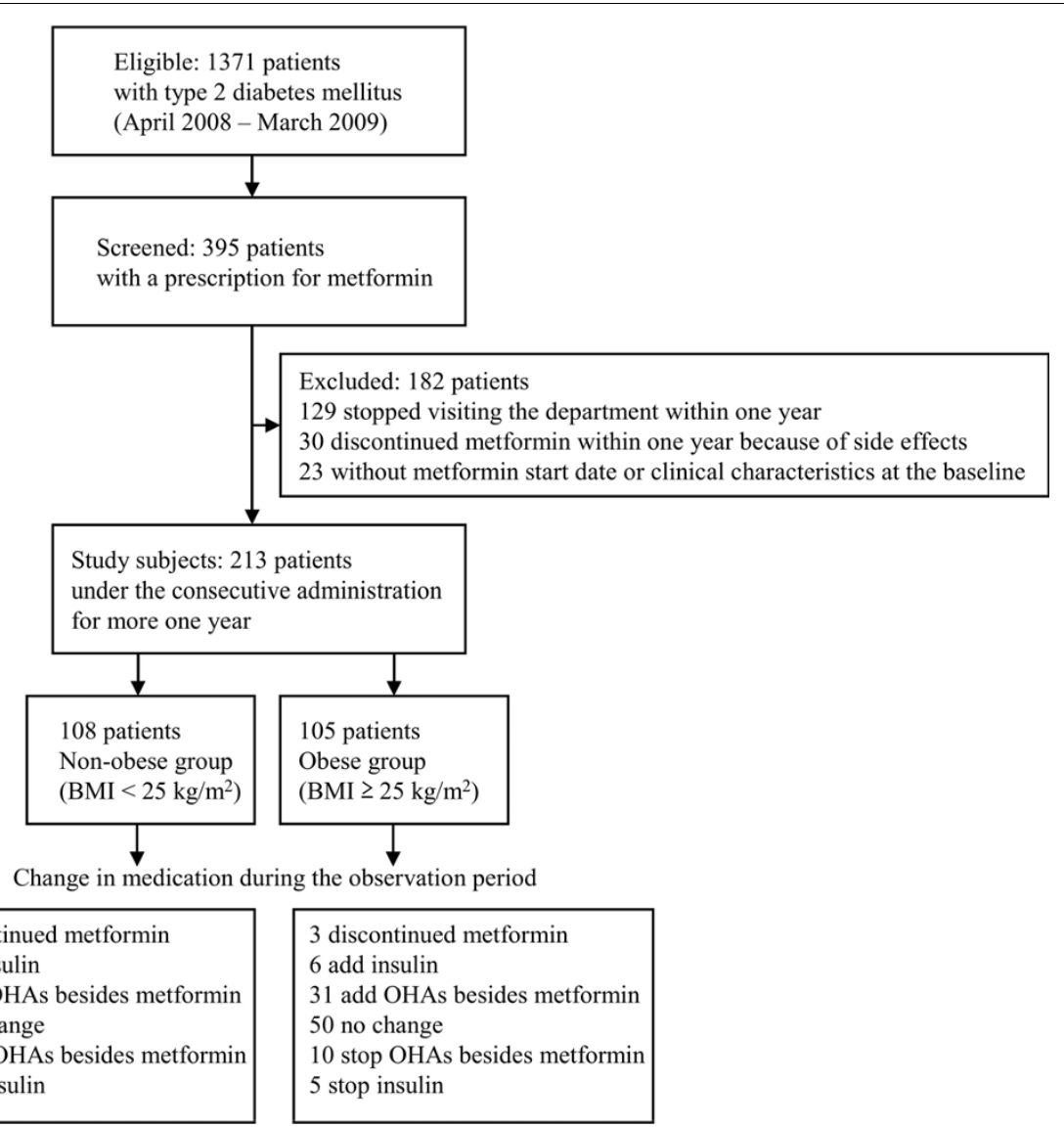

Figure 1 Flow diagram of the population cohort. BMI: body mass index. OHAs: oral hypoglycemic agents. 
respectively. Hypertension was defined as a systolic blood pressure $\geq 140 \mathrm{mmHg}$ and/or a diastolic blood pressure $\geq 90 \mathrm{mmHg}$. The participants currently using antihypertensive medications were also classified as positive for hypertension. Serum total cholesterol, LDLcholesterol, and HDL-cholesterol concentrations were measured with TBA-200 FR NEO using Determiner L TC II, Determiner L LDL-C, and Determiner L HDL-C instruments (Kyowa Medex Co., Ltd., Tokyo, Japan). Hyperlipidaemia was defined by serum concentrations of total cholesterol levels $\geq 5.7 \mathrm{mmol} / \mathrm{L}$, LDL-cholesterol levels $\geq 3.6 \mathrm{mmol} / \mathrm{L}$, or as patients who were already undergoing treatment with lipid-lowering agents. The triglyceride concentrations were not investigated in this study because fasting blood samples could not always be obtained for measurements. HbA1c levels were determined with a high performance liquid chromatography method using an automated HLC-723G7 analyzer (Tosoh Corporation, Tokyo, Japan) and calibrated by the Japan Diabetes Society (JDS) standard calibrators. The eGFR was calculated using the formula reported by Matsuo et al [15]. This equation originated from the MDRD study group [16] arranged for Japanese individuals, and it is recommended by the Japanese Society of Nephrology: eGFR $\left(\mathrm{mL} / \mathrm{min} / 1.73 \mathrm{~m}^{2}\right)=194 \times \mathrm{Scr}^{-1.094}$ $\times \mathrm{Age}^{-0.287} \times 0.739$ (if female).

\section{Statistical analysis}

An analysis of variance (ANOVA) and the $\chi^{2}$ test were used for between-group comparisons of the continuous and categorical variables, respectively. A paired $t$-test was conducted to determine whether there were any differences in the dose of metformin, HbA1c levels or BMI during the observation period compared to the baseline values. Pearson's univariate regression was performed to determine whether there was any association of reduced HbA1c with other clinical parameters. The odds ratio (OR) and respective $95 \%$ confidence interval (95\% CI) were determined to examine the strength of the relationship between the requirement of additional glucose-lowering agents (OHAs or insulin) and the clinical parameters based on a multiple logistic regression analysis. Differences with $P$-values of less than 0.05 (two-tailed) were considered to be statistically significant. The statistical software package JMP, version 8.0 (SAS Institute, Cary, NC, USA), was used to perform all of the analyses.

\section{Results}

Table 1 shows the baseline characteristics of the study patients. The age and the duration of diabetes mellitus were significantly higher and longer in the non-obese group than in the obese group. There was a greater frequency of patients without any antidiabetic agents prior to metformin administration in the obese group (31\%)
Table 1 Baseline characteristics of the patients

\begin{tabular}{|c|c|c|c|}
\hline & $\begin{array}{c}\begin{array}{c}\text { Non- } \\
\text { obese }\end{array} \\
(n=108)\end{array}$ & $(n=105)$ & $P$ \\
\hline Body mass index $\left(\mathrm{kg} / \mathrm{m}^{2}\right)$ & $22.7 \pm 1.7$ & $28.4 \pm 2.9$ & $<0.01$ \\
\hline Age (years) & $64 \pm 7$ & $59 \pm 10$ & $<0.01$ \\
\hline Men $(\%)$ & 49 & 52 & 0.63 \\
\hline Duration of diabetes mellitus (years) & $12 \pm 9$ & $8 \pm 7$ & $<0.01$ \\
\hline Current plus past smoking (\%) & 62 & 67 & 0.59 \\
\hline Current drinkers (\%) & 58 & 60 & 0.79 \\
\hline \multicolumn{4}{|l|}{$\begin{array}{l}\text { Therapeutic method for diabetes } \\
\text { mellitus }\end{array}$} \\
\hline Diet only/OHAs/Insulin (\%) & $16 / 67 / 18$ & $31 / 48 / 21$ & $<0.01$ \\
\hline Systolic blood pressure (mmHg) & $132 \pm 16$ & $137 \pm 16$ & 0.01 \\
\hline Diastolic blood pressure (mmHg) & $78 \pm 10$ & $81 \pm 11$ & 0.02 \\
\hline Hypertension (\%) & 61 & 87 & $<0.01$ \\
\hline Total cholesterol (mmol/L) & $5.3 \pm 1.1$ & $5.3 \pm 1.1$ & 0.71 \\
\hline LDL cholesterol (mmol/L) & $2.9 \pm 0.7$ & $3.1 \pm 0.8$ & $<0.01$ \\
\hline HDL cholesterol (mmol/L) & $1.6 \pm 0.5$ & $1.4 \pm 0.3$ & 0.09 \\
\hline Hyperlipidaemia (\%) & 81 & 84 & 0.52 \\
\hline Haemoglobin A1c (\%) & $8.1 \pm 2.0$ & $8.1 \pm 1.7$ & 0.90 \\
\hline Uric acid ( $\mu \mathrm{mol} / \mathrm{L})$ & $282 \pm 84$ & $302 \pm 79$ & 0.10 \\
\hline Serum creatinine $(\mu \mathrm{mol} / \mathrm{L})$ & $67 \pm 17$ & $67 \pm 15$ & 0.71 \\
\hline Estimated GFR (mL/min/1.73 m²) & $72.6 \pm 17.3$ & $\begin{array}{l}75.5 \pm \\
17.7\end{array}$ & 0.25 \\
\hline Initial dose of metformin (mg/day) & $556 \pm 110$ & $566 \pm 116$ & 0.49 \\
\hline
\end{tabular}

OHAs: oral hypoglycemic agents, GFR: glomerular filtration rate

than in the non-obese group (16\%). The blood pressure levels, the prevalence of hypertension and serum LDLcholesterol concentrations were significantly higher in the obese patients than in the non-obese patients. The $\mathrm{HbA1c}$ levels and the initial dose of metformin were not different between the two groups.

During the observation period, HbA1c levels were significantly decreased compared with the baseline levels (Figure 2). The course of HbA1c was equal between the non-obese and the obese groups. HbA1c was reduced by $1.2 \%$ and $1.1 \%$ at 12 months, $0.9 \%$ and $0.9 \%$ at 24 months, $0.8 \%$ and $1.0 \%$ at 36 months in the nonobese and obese groups, respectively. Although the dose of metformin required to control the blood glucose levels gradually increased during the observation period (Figure 3), it was significantly lower in the non-obese group $(598 \pm 132 \mathrm{mg} /$ day at 12 months, $614 \pm 138 \mathrm{mg} /$ day at 24 months and $677 \pm 184 \mathrm{mg} /$ day at 36 months) than in the obese group $(678 \pm 169 \mathrm{mg} /$ day at 12 months, $696 \pm 170 \mathrm{mg}$ /day at 24 months and $724 \pm 117$ $\mathrm{mg} /$ day at 36 months). However, the dose of metformin after body weight correction was significantly higher in the non-obese group than in the obese group over the course of the observation periods (Figure 3 ). The values of BMI were not significantly different through all periods in the two groups (Figure 2). 


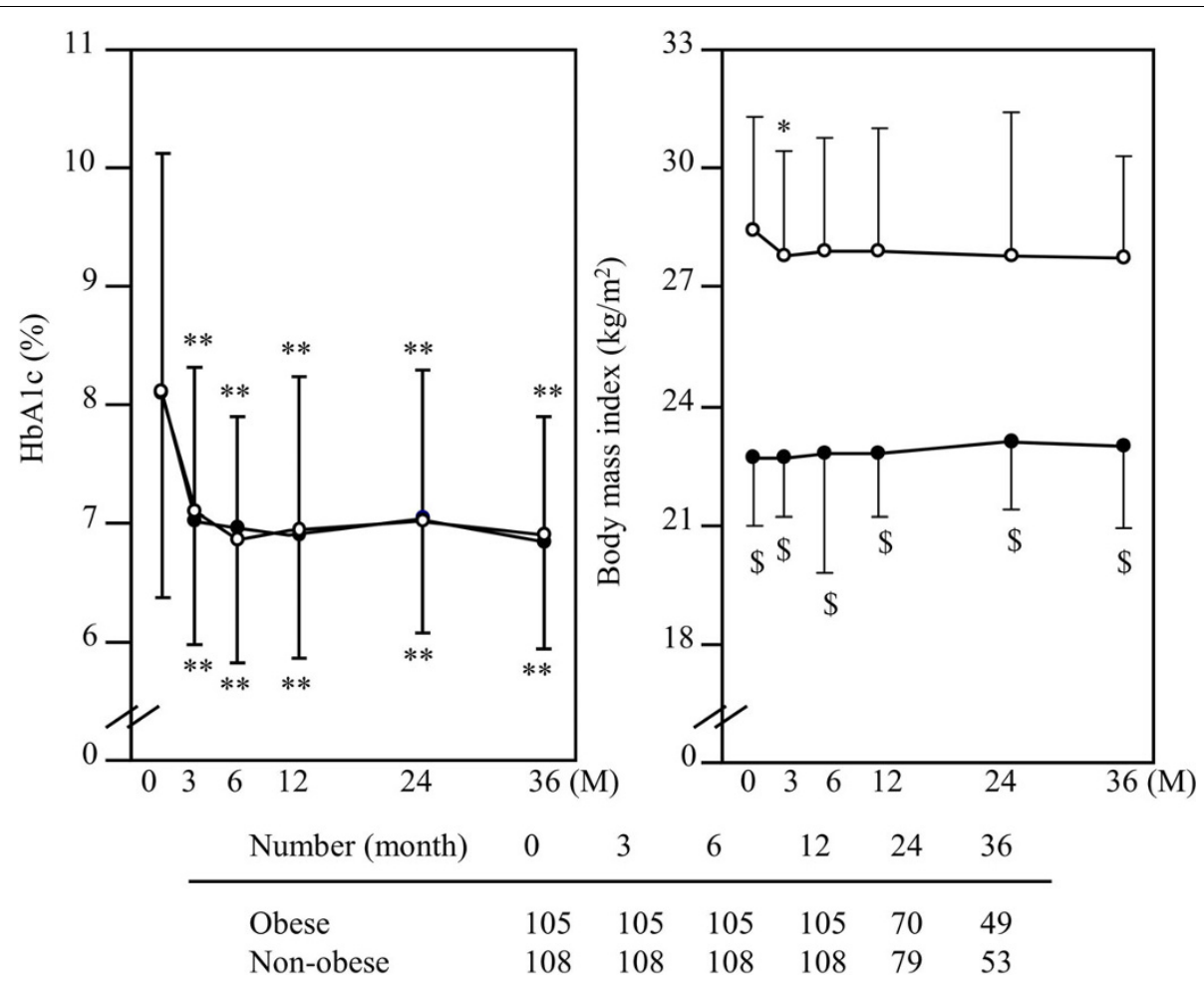

Figure 2 Changes of HbA1c levels and body mass index in obese and non-obese patients with type 2 diabetes mellitus. Data represent the mean \pm SD. Open and closed circles represent the values in obese and non-obese individuals, respectively. ${ }^{*} P<0.05$ and ${ }^{* *} P<0.01$ vs. 0 $M$ (initiation of metformin). ${ }^{\$} P<0.01$ vs. obese.

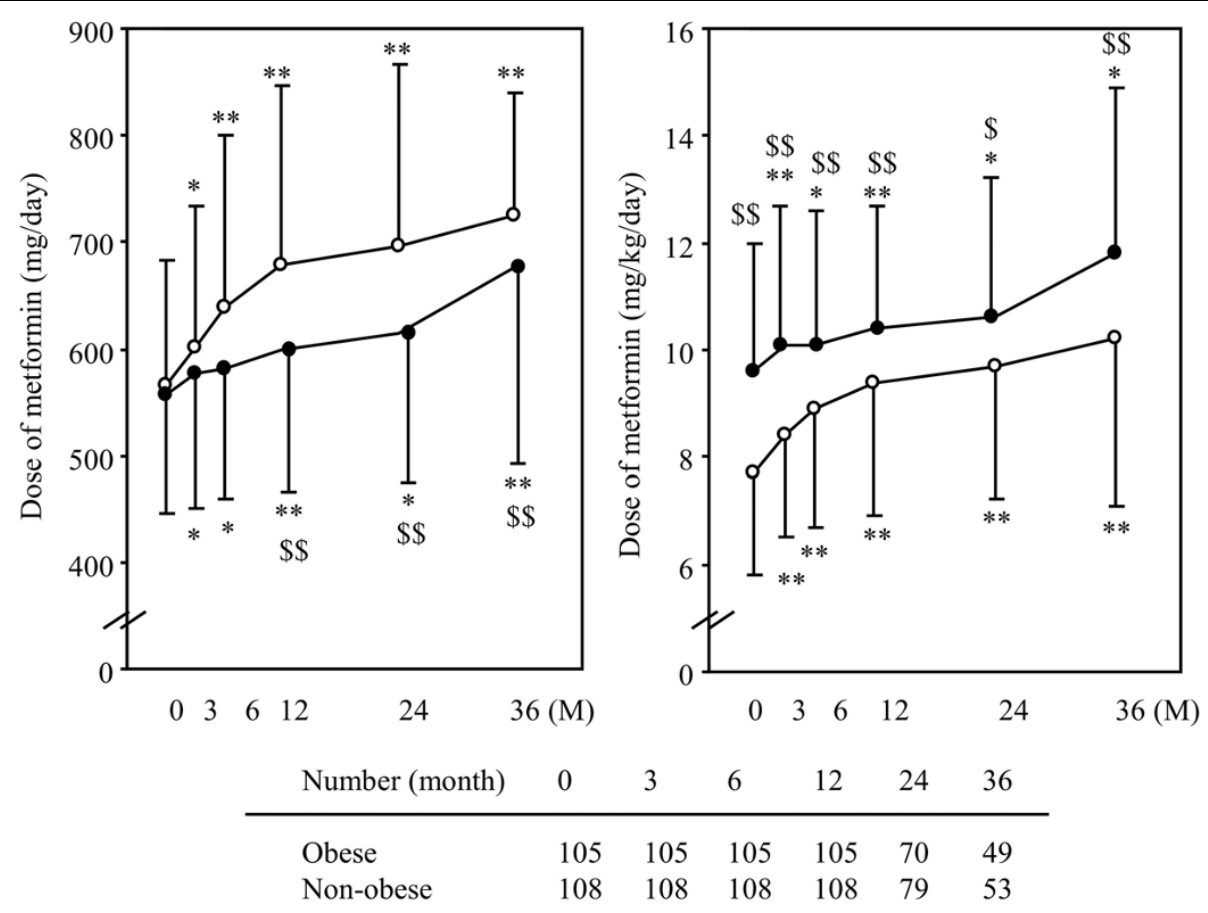

Figure 3 Changes in the dose of metformin and those corrected by the patient's body weight. Data represent the mean \pm SD. Open and closed circles represent the values in obese and non-obese individuals, respectively. ${ }^{*} P<0.05$ and ${ }^{* *} P<0.01$ vs. $0 \mathrm{M}$ (initiation of metformin). $P<0.05$ and $\$ \$ P<0.01$ vs. obese. 
The correlations of the clinical parameters to the reduction of HbA1c are shown in Table 2. Only HbA1c levels at the baseline time were significantly correlated with the reduction in each time of the observation period, although the duration of diabetes mellitus only showed a correlation at 1 year after the initiation of metformin.

None of the patients were omitted from the study due to the side effects induced by metformin. Three patients discontinued metformin because of the progression of renal dysfunction in both obese and non-obese groups during the observation period (Figure 1). After starting the metformin treatment, three patients $(3 \%)$ in the non-obese group and 6 patients (6\%) in the obese group were introduced onto insulin therapy. Additional OHAs besides metformin were required in $43(40 \%)$ of the non-obese and $31(30 \%)$ of the obese patients. Fortyeight (44\%) and 50 (48\%) patients required no additional antidiabetic agents (other OHAs or insulin) or a reduction of the treatment in the non-obese and obese groups, respectively. The other OHAs besides metformin were either reduced or discontinued in $10(9 \%)$ of non-obese and $10(10 \%)$ of obese patients. One (1\%) of the non-obese and $5(5 \%)$ of the obese patients no longer required insulin therapy. The proportion of the changes in these treatment components was not significantly different between the non-obese and obese groups based on a $\chi^{2}$ test. Table 3 shows the odds ratio for the requirement of additional agents (other OHAs or insulin) in the non-obese and obese patients with type 2 diabetes mellitus according to a logistic regression analysis adjusted by age, HbA1c at baseline and the therapeutic method (diet $=0$ and oral hypoglycemic agents plus insulin $=1$ ). The treatment using additional

Table 2 HbA1c reduction determinants by correlation coefficient

\begin{tabular}{lcccccc}
\hline & \multicolumn{2}{c}{$\mathbf{1}$ year } & \multicolumn{2}{c}{$\mathbf{2}$ years } & \multicolumn{2}{c}{$\mathbf{3}$ years } \\
\cline { 2 - 7 } & $\boldsymbol{r}$ & $\boldsymbol{P}$ & $\boldsymbol{r}$ & $\boldsymbol{P}$ & $\boldsymbol{r}$ & $\boldsymbol{P}$ \\
\hline Sex & 0.06 & 0.42 & -0.07 & 0.42 & -0.15 & 0.14 \\
Age & 0.11 & 0.60 & -0.02 & 0.79 & 0.14 & 0.16 \\
Duration of diabetes & 0.24 & $<0.01$ & 0.16 & 0.10 & 0.15 & 0.21 \\
Body mass index & -0.04 & 0.54 & -0.04 & 0.61 & -0.10 & 0.34 \\
HbA1c at baseline & -0.81 & $<0.01$ & -0.73 & $<0.01$ & -0.82 & $<0.01$ \\
Hypertension & -0.09 & 0.19 & -0.01 & 0.93 & -0.01 & 0.91 \\
Hyperlipidaemia & -0.02 & 0.75 & -0.04 & 0.65 & 0.23 & 0.47 \\
Estimated GFR & 0.03 & 0.69 & 0.04 & 0.63 & 0.04 & 0.67 \\
Therapeutic method & 0.13 & 0.07 & 0.17 & 0.10 & 0.11 & 0.27 \\
Initial dose of metformin & -0.11 & 0.11 & 0.01 & 0.94 & -0.03 & 0.75 \\
Metformin dose at 1 year & -0.07 & 0.32 & 0.08 & 0.36 & -0.01 & 0.86 \\
\hline
\end{tabular}

GFR: glomerular filtration rate.

Sex $(\operatorname{man}=1$, woman $=2)$, therapeutic method (diet $=1$, oral hypoglycemic agents $=2$, insulin $=3$ ), hypertension (absent $=0$, present $=1$ ) and hyperlipidaemia (absent $=0$, present $=1$ ) were replaced with a number.
Table 3 Odds ratios for the requirement of additional agents in the non-obese and obese patients with type 2 diabetes mellitus determined by a logistic regression analysis

\begin{tabular}{llll}
\hline & Wald $\boldsymbol{\chi}^{\mathbf{2}}$ score & OR $(\mathbf{9 5} \% \mathbf{C I})$ & $\boldsymbol{P}$ \\
\hline Non-obese & & & \\
\hline Age & 1.52 & $0.91(0.91-1.02)$ & 0.22 \\
HbA1c at baseline & 0.08 & $0.97(0.77-1.19)$ & 0.78 \\
$\quad$ Therapeutic method & 5.76 & $0.24(0.07-0.74)$ & 0.02 \\
\hline Obese & & & \\
\hline Age & 0.32 & $1.01(0.97-1.06)$ & 0.57 \\
HbA1c at baseline & 4.27 & $1.31(1.02-1.71)$ & 0.04 \\
$\quad$ Therapeutic method & 5.12 & $0.35(0.14-0.87)$ & 0.02 \\
\hline
\end{tabular}

Adjusted by age, HbA1c at the baseline, and the therapeutic method (diet, oral hypoglycemic agents + insulin).

OR: odds ratio, $\mathrm{Cl}$ : confidence interval.

agents was significantly more frequent in the individuals with diet therapy alone at baseline than in those using other glucose-lowering drugs in both non-obese and obese patients. The use of additional agents was also required more frequently in the patients showing high HbA1c levels at the baseline among the obese group. The model adjusted by the duration of diabetes mellitus, HbA1c at baseline and the therapeutic method showed similar statistical results (date are not shown).

Figure 4 shows the changes of HbA1c levels among the subgroups as divided by therapeutic methods (diet only, OHAs and insulin) at the baseline in the non-obese and obese patients with type 2 diabetes mellitus. Although HbA1c was significantly lower in the subgroup using OHAs at the baseline time, the changes in HbA1c levels during the observation period were not significantly different among the three subgroups in the non-obese patients. On the other hand, HbA1c was significantly higher in the subgroups using OHAs or insulin at 24 and 36 months in the obese patients.

To possibly minimize any interference caused by known changes in the other OHAs or insulin during the observation period, the changes in HbA1c levels were compared in the patients who required no additional antidiabetic agents or reduction in treatment between the non-obese $(n=48)$ and obese groups $(n=50)$. The course of HbA1c levels was not significantly different between the two groups (Figure 5). The reduction of HbA1c was $1.1 \%$ and $0.9 \%$ at 12 months, $1.1 \%$ and $0.8 \%$ at 24 months, $1.2 \%$ and $1.0 \%$ at 36 months in the nonobese and obese groups, respectively.

\section{Discussion}

This study demonstrated the long-term beneficial effect of metformin therapy in non-obese (BMI $<25 \mathrm{~kg} / \mathrm{m}^{2}$ ) patients with type 2 diabetes mellitus. Metformin induced a significant reduction in the HbA1c levels in 


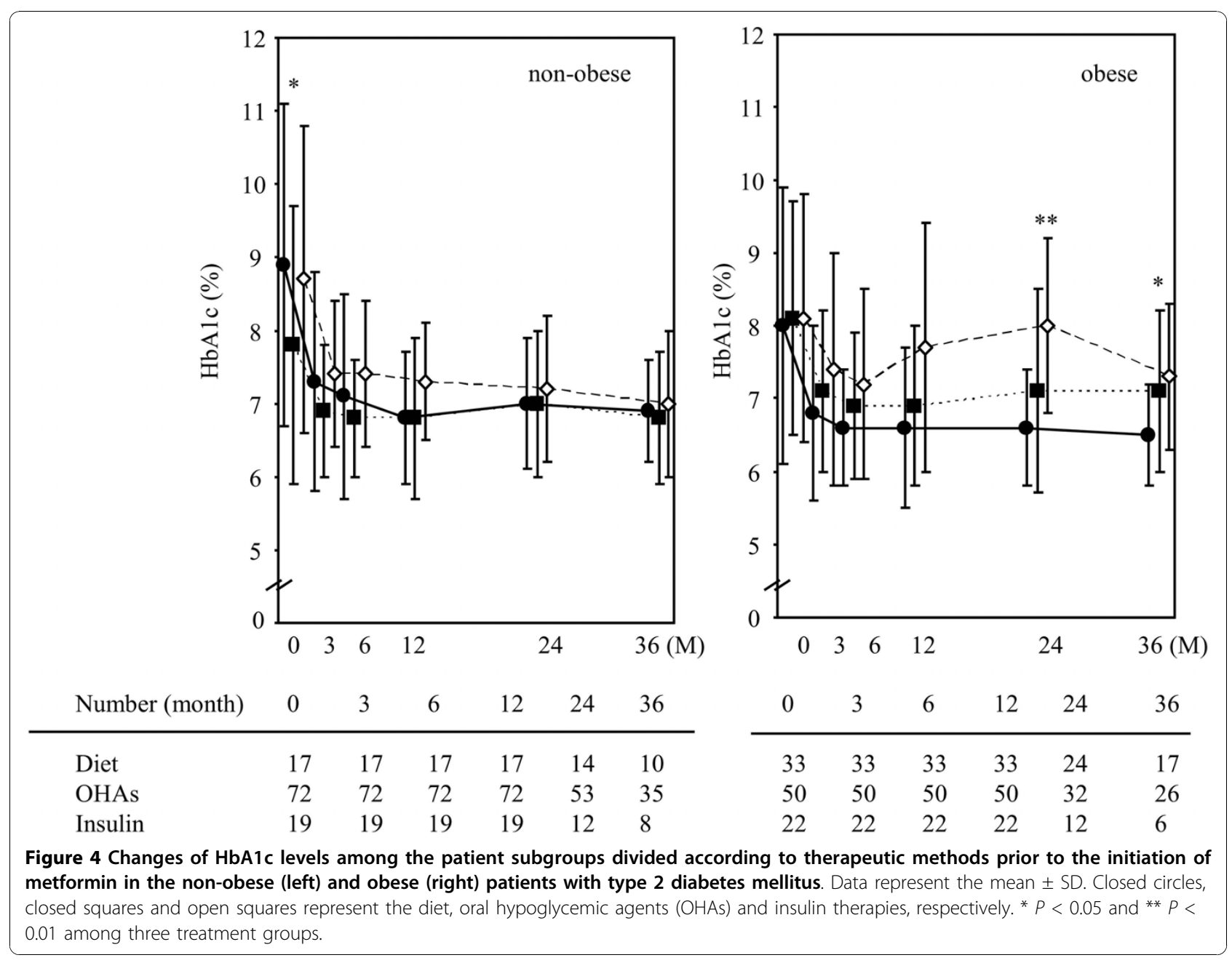

non-obese patients. This effect was similar between nonobese and obese subjects who needed no additional antidiabetic agents or a reduction in other treatments during the observation period (Figure 5). Furthermore, similar glucose-lowering effects were obtained among the non-obese subgroups divided by the therapeutic methods prior to the initiation of metformin (Figure 4).

Although several studies have shown the advantage of metformin in non-obese diabetic patients [6-12], the observation periods were relatively short. Kaku et al. reported that the reduction of $\mathrm{HbA} 1 \mathrm{c}$ by metformin was not different between 303 non-obese $(0.9 \%$, BMI $<25$ $\left.\mathrm{kg} / \mathrm{m}^{2}\right)$ and 300 obese $\left(1.0 \%\right.$, BMI $\left.\geq 25 \mathrm{~kg} / \mathrm{m}^{2}\right)$ Japanese patients with type 2 diabetes mellitus according to a prospective study for 12 months [6]. Hosokawa et al. retrospectively showed a significant $0.79 \%$ reduction in HbA1c levels in 58 patients with a BMI $\leq 22 \mathrm{~kg} / \mathrm{m}^{2}$, $0.81 \%$ in 81 patients with $22<\mathrm{BMI}<25 \mathrm{~kg} / \mathrm{m}^{2}$, and $0.73 \%$ in 136 patients with $25 \mathrm{~kg} / \mathrm{m}^{2} \leq$ BMI 12 months after the initiation of metformin for type 2 diabetes mellitus [7]. Clarke and Campbell reported that metformin monotherapy $(n=98)$ was equally effective as chlorpropamide $(n=91)$, one of the sulfonylurea agents, on blood glucose control without HbA1c measurements, and that metformin was superior in the body weight control in non-obese patients with type 2 diabetes mellitus according to a prospective study for 1 year [8]. Yajima et al. demonstrated the metformin administration at a dose of 500-750 mg/day to be more effective in non-obese patients $\left(n=22\right.$, mean BMI was $\left.25.6 \mathrm{~kg} / \mathrm{m}^{2}\right)$ with type 2 diabetes mellitus than in those treated at a dose of 150-300 mg/day of acarbose, an $\alpha$-glucosidase inhibitors, in a crossover study conducted with 3-months treatment periods [9]. Lund et al. described that the glycemic regulation was equivalent between metformin and repaglinide, an insulin secretagogue, in a 4-month crossover trial in 96 non-obese (BMI $\leq 27 \mathrm{~kg}$ / $\mathrm{m}^{2}$ ) European patients with type 2 diabetes mellitus [10]. They also reported that the effect of metformin $(n=52)$ and repaglinide $(n=49)$ was not significantly different when combined with insulin for the treatment of non-obese patients, according to a randomized 


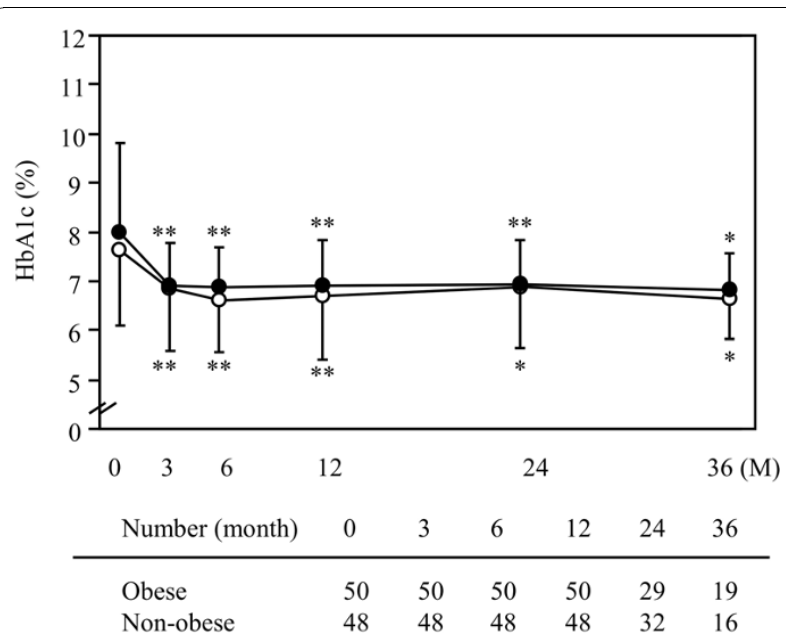

Figure 5 Changes in $\mathrm{HbA} 1 \mathrm{c}$ levels in patients who required no additional antidiabetic agents or a reduction in treatment. Data represent the mean \pm SD. Open and closed circles represent the values in obese and non-obese individuals, respectively. ${ }^{*} P<0.05$ and ${ }^{*} P<0.01$ vs. $0 \mathrm{M}$ (initiation of metformin).

prospective study for 12 months [11]. Donnelly et al. showed the glucose-lowering effect of metformin to be very similar between the non-obese and obese patients with type 2 diabetes mellitus according to a study covering a period ranging from 3-12 months [12]. Ong at al. revealed that metformin was efficacious in non-obese subjects with type 2 diabetes mellitus $(n=136)$ according to a retrospective analysis of 16 years [17]. This is the first report to show the long-term effect of metformin among obese and non-obese patients with type 2 diabetes mellitus. Although the definition of non-obese was a BMI of less than $30 \mathrm{~kg} / \mathrm{m}^{2}$, their investigation included $7 \%(n=27)$ of individuals with a BMI $<25 \mathrm{~kg} /$ $\mathrm{m}^{2}$ in their study subjects.

The glucose-lowering effect of metformin might be overestimated in the present study because the HbA1c levels were obtained from patients who had used metformin for more than one year. Individuals who stopped this treatment because of a lack of efficacy or side effects within one year were excluded. However, this limitation could be similar between the non-obese and obese groups.

The effects of OHAs and insulin gradually decrease as the treatment periods become longer $[2,13]$. Successive reduction of insulin from the pancreatic $\beta$-cells in patients with type 2 diabetes mellitus is considered to be a major factor for this attenuation of the drug effects [18-20]. The present study showed that a gradual increase of the metformin was required to control blood glucose levels during the observation period. However, the metformin dose increase was significantly lower in non-obese patients than in obese patients. Specifically, it may be possible to treat non-obese diabetic patients using a lower dose of metformin compared with obese patients. Donnelly et al. also reported that metformin was more effective in type 2 diabetic patients with a lower BMI [12]. However, the dose of metformin was higher in the non-obese patients than in the obese patients after the body weight correction in this study (Figure 3). It seems to be a paradox that a lower total dose of metformin while at the same time a higher dose corrected for body weight was found in the non-obese patients. In the present study, the duration of diabetes mellitus was significantly longer in the non-obese patients than in the obese patients. Furthermore, other glucose-lowering therapies, including OHAs and insulin were more frequently administered to the non-obese than to the obese patients. This underscored a potentially more severe stage of disease in the non-obese rather than in the obese patients. It is therefore possible that the endogenous insulin secretion was more strongly impaired in the non-obese than in the obese group, while the insulin resistance caused by, for example, visceral fat also was less in the non-obese than in the obese subjects of the present study. Although the parameters indicating insulin resistance and endogenous insulin secretion, such as serum or urine $\mathrm{C}$-peptide concentration, were not evaluated in this study, the lower insulin resistance and secretion in the non-obese patients might have caused an increase in the requirement per body weight of metformin.

The levels of HbA1c were maintained within less than $7 \%$ during the 3-year observation period of this study. Additional OHAs or insulin treatment was necessary in 46 (43\%) non-obese and 37 (35\%) obese patients. It may also be caused by the attenuation of endogenous insulin secretion [18-20]. However, approximately half of patients required no addition or reduction of other treatments after the initiation of metformin in either group. Although the present study was not performed using metformin monotherapy, our results clinically demonstrated the advantage of metformin either in combination with the other antidiabetic agents or as a single therapy without any change in the treatment.

Metformin appears to maintain the glucose-lowering effect even after the observation period of this study had concluded, because the dose in the non-obese group was limited to $677 \mathrm{mg}$ at 36 months of this study. The worsening in glycemic control over time observed in many patients with type 2 diabetes mellitus can probably be attenuated by increasing the dose of metformin because metformin reduces the HbA1c levels in a doserelated manner [21]. Metformin is a tolerable drug for patients if the dosage of the first year is completed, because no patients were omitted from this study due to side effects caused by metformin. 
The results of this study have several limitations that must be considered. First, the findings are inherently limited by an inability to eliminate the effects caused by the other glucose-lowering agents, because our investigation included patients receiving a combination therapy of metformin and other antidiabetic drugs. It is difficult to study the long-term course of metformin monotherapy because of the attenuation of the effect as time passes [2,13]. Only 11 (3 non-obese and 8 obese) patients were treated with metformin monotherapy during the entire period, thus making it impossible to determine the significance of the therapy in these patients. To possibly minimize the interference caused by known changes in the other OHAs or insulin, the changes in HbA1c levels were compared in the patients who required no additional antidiabetic agents or reduction in treatment between the non-obese and obese groups (Figure 5). Although the change in the insulin dose is considered to be important among the patients receiving insulin treatment at baseline, this factor was not investigated in the present study. We therefore cannot exclude the possibility that some differences in the need for increasing the insulin doses between the nonobese and obese group of patients confounded our data with respect to the efficacy of metformin treatment during this study. This factor could also be a confounder even in those patients with no known (other) changes in the treatment. Second, this investigation is a retrospective study without the obvious criteria for the changes in treatment methods. Because the optimization of the metformin dose and the coordination of the treatment were dependent on each patient's physician, we considered that errors on the effects of metformin could have occurred. Third, the present study did not include a control group with a different treatment regimen, such as insulin secretagogues. Therefore, it is unknown whether the observed similarities and differences between the non-obese and obese patients were specific for these groups and/or specific effects of metformin therapy. Fourth, the associations of metformin with diabetic vascular events were not examined in this study. Furthermore, the risk factors for cardiovascular diseases such as blood pressure and serum lipid profile were not analyzed during the observation period because various antihypertensive agents and statins were used. The results of this study demonstrated the glucose-lowering effect of metformin in a group of non-obese patients with type 2 diabetes mellitus who continued such treatment for at least 1 year. Therefore, investigations to evaluate the effect of metformin therapy to potentially reduce the risk of developing diabetic complications also in non-obese patients with type 2 diabetes mellitus should be considered in the future.

\section{Declaration of competing interests}

The authors declare that they have no competing interests.

\section{Acknowledgements}

The authors thank Ms. Sakura Yamamoto and Ms. Tomoko Koyanagi in the secretarial section of Edogawa Hospital for their valuable helps with the data collection.

\section{Authors' contributions}

HIt and HIs contributed to the design of the study, analysis and interpretation of data, and drafting of the manuscript. YT, SA, MA, MM and MT contributed to interpretation of the data and critical revision of the manuscript. All authors have given their final approval of the submitted version of the manuscript.

Received: 18 August 2010 Accepted: 12 November 2010

Published: 12 November 2010

\section{References}

1. Nathan DM, Buse JB, Davidson MB, et al: Medical management of hyperglycaemia in type 2 diabetes mellitus: a consensus algorithm for the initiation and adjustment of therapy: a consensus statement from the American Diabetes Association and the European Association for the Study of Diabetes. Diabetologia 2009, 52:17-30.

2. UK Prospective Diabetes Study (UKPDS) Group: Effect of intensive bloodglucose control with metformin on complications in overweight patients with type 2 diabetes (UKPDS 34). Lancet 1998, 352:854-865.

3. United Kingdom Prospective Diabetes Study Group: United Kingdom Prospective Diabetes Study 24: a 6-year, randomized, controlled trial comparing sulfonylurea, insulin, and metformin therapy in patients with newly diagnosed type 2 diabetes that could not be controlled with diet therapy. Ann Intern Med 1998, 128:165-175.

4. Deurenberg P, Deurenberg-Yap M, Guricci S: Asians are different from Caucasians and from each other in their body mass index/body fat per cent relationship. Obes Rev 2002, 3:141-146.

5. Sone $H$, Tanaka S, limuro $\mathrm{S}$, et al: Components of metabolic syndrome and their combinations as predictors of cardiovascular disease in Japanese patients with type 2 diabetes. Implications for improved definition. Analysis from Japan Diabetes Complications Study (JDCS). J Atheroscler Thromb 2009, 16:380-387.

6. Kaku K, Tajima N, Kawamori K: Melbin Observation Research (MORE) study of metformin therapy in patients with type 2 diabetes mellitus. $J$ Japan Diab Soc 2006, 49:325-331.

7. Hosokawa K, Meguro S, Funae $O$, et al: Clinical effects of metformin with nonobese type 2 diabetes. J Japan Diab Soc 2009, 52:1-6.

8. Clarke BF, Campbell IW: Comparison of metformin and chlorpropamide in non-obese, maturity-onset diabetics uncontrolled by diet. $\mathrm{Br}$ Med J 1977, 2(6102):1576-1578.

9. Yajima K, Shimada A, Hirose H, Kasuga A, Saruta T: "Low dose" metformin improves hyperglycemia better than acarbose in type 2 diabetics. Rev Diabet Stud 2004, 1:89-94.

10. Lund SS, Tarnow L, Stehouwer CD, et al: Targeting hyperglycaemia with either metformin or repaglinide in non-obese patients with type 2 diabetes: results from a randomized crossover trial. Diabetes Obes Metab 2007, 9:394-407.

11. Lund SS, Tarnow L, Frandsen M, et al: Combining insulin with metformin or an insulin secretagogue in non-obese patients with type 2 diabetes: 12 month, randomised, double blind trial. BMJ 2009, 339:b4324.

12. Donnelly LA, Doney AS, Hattersley AT, Morris AD, Pearson ER: The effect of obesity on glycaemic response to metformin or sulphonylureas in Type 2 diabetes. Diabet Med 2006, 23:128-133.

13. Kahn SE, Haffner SM, Heise MA, et al: Glycemic durability of rosiglitazone, metformin, or glyburide monotherapy. N Engl J Med 2006, 355:2427-2443.

14. Japan Diabetes Society: Treatment Guide for Diabetes 2007 Tokyo: Bunkodo; 2007.

15. Matsuo S, Imai E, Horio M, et al: Revised equations for estimated GFR from serum creatinine in Japan. Am J Kid Dis 2009, 53:982-992. 
16. Coresh J, Astor BC, Greene T, Eknoyan G, Levey AS: Prevalence of chronic kidney disease and decreased kidney function in the adult US population. Am J Kid Dis 2003, 41:1-12.

17. Ong CR, Molyneaux LM, Constantino MI, Twigg SM, Yue DK: Long-term efficacy of metformin therapy in nonobese individuals with type 2 diabetes. Diabetes Care 2006, 29:2361-2364.

18. U.K Prospective Diabetes Study Group: U.K. prospective diabetes study 16 . Overview of 6 years' therapy of type II diabetes: a progressive disease. Diabetes 1995, 44:1249-1258.

19. Levy J, Atkinson AB, Bell PM, McCance DR, Hadden DR: Beta-cell deterioration determines the onset and rate of progression of secondary dietary failure in type 2 diabetes mellitus: the 10-year follow-up of the Belfast Diet Study. Diabet Med 1998, 15:290-296.

20. Defronzo RA: Banting Lecture. From the triumvirate to the ominous octet: a new paradigm for the treatment of type 2 diabetes mellitus. Diabetes 2009, 58:773-795.

21. Garber AJ, Duncan TG, Goodman AM, Mills DJ, Rohlf JL: Efficacy of metformin in type II diabetes: results of a double-blind, placebocontrolled, dose-response trial. Am J Med 1997, 103:491-497.

doi:10.1186/1743-7075-7-83

Cite this article as: Ito et al.: Long-term effect of metformin on blood glucose control in non-obese patients with type 2 diabetes mellitus. Nutrition \& Metabolism 2010 7:83.

\section{Submit your next manuscript to BioMed Central} and take full advantage of:

- Convenient online submission

- Thorough peer review

- No space constraints or color figure charges

- Immediate publication on acceptance

- Inclusion in PubMed, CAS, Scopus and Google Scholar

- Research which is freely available for redistribution

Submit your manuscript at www.biomedcentral.com/submit
C Biomed Central 